\title{
Visual Perceptual Skills in Very Preterm Children : \\ Developmental Course and Associations With Neural Activation
}

\section{PIPARI Study Grp}

2020-08

PIPARI Study Grp , Lind, A , Parkkola , R , Laasonen , M , Vorobyev , V \& Haataja , L 2020

, ' Visual Perceptual Skills in Very Preterm Children : Developmental Course and Associations With Neural Activation ' , Pediatric Neurology , vol. 109 , pp. 72-78 . https://doi.org/10.1016/j.pediatrneu

http://hdl.handle.net/10138/329544

https://doi.org/10.1016/j.pediatrneurol.2020.04.012

cc_by_nc_nd

acceptedVersion

Downloaded from Helda, University of Helsinki institutional repository.

This is an electronic reprint of the original article.

This reprint may differ from the original in pagination and typographic detail.

Please cite the original version. 


\section{Visual Perceptual Skills in Very Preterm Children: Developmental Course and}

\section{Associations with Neural Activation}

Annika Lind $^{\text {ab }}$, Riitta Parkkola ${ }^{\mathrm{c}}$, Marja Laasonen ${ }^{\mathrm{d}}$, Victor Vorobyev ${ }^{\mathrm{c}}$, Leena Haataja ${ }^{\mathrm{e}}$ on behalf of the PIPARI Study Group

${ }^{a}$ Department of Psychology, University of Turku, Turku, Finland

${ }^{\mathrm{b}}$ Turku Institute for Advanced Studies (TIAS), University of Turku, Turku, Finland

${ }^{\mathrm{c}}$ Department of Radiology, Turku University and Turku University Hospital, Turku, Finland

${ }^{\mathrm{d}}$ Department of Speech and Language Pathology, University of Turku, Turku, Finland, Department of Otorhinolaryngology and Phoniatrics, Head and Neck Surgery, Helsinki University Hospital and University of Helsinki, Helsinki, Finland and Department of Psychology and Logopedics, University of Helsinki, Helsinki, Finland

e University of Helsinki and Helsinki University Hospital, Children's Hospital and Pediatric Research Center, Helsinki, Finland

Corresponding author: Annika Lind, annika.lind@utu.fi, postal address Department of Psychology, University of Turku, 20014 Turun yliopisto, Finland

\section{Declarations of interest: none}

Funding: This work was supported by governmental funding for research in university hospitals ('ERVA' funding); Foundation for Paediatric Research; and Turku Institute for Advanced Studies

Word count: 4167

Running title: Visual perception and prematurity 


\section{Abstract}

Objective: To examine how non-verbal skills at five years of age relate to visual perception and brain activation during visual perception tasks at twelve years of age in very preterm subjects without visual or other neurodevelopmental impairments or major brain pathologies. Methods: At five years of age, 36 prematurely born (birth weight $\leq 1500$ grams and/or gestational age $<32$ weeks) and 31 term-born control children were assessed with the nonverbal subtests of the Wechsler Preschool and Primary Scale of Intelligence-Revised and the NEPSY II. At twelve years of age, the same children were re-assessed with tasks from the Motor-Free Visual Perception Test, Third Edition during functional magnetic resonance imaging.

Results: Test performance at five years of age was significantly poorer in the very preterm group than the controls, but at twelve years of age performance was similar in both groups. In the very preterm group, better non-verbal skills at five years of age were significantly associated with stronger neural activation during the visual perception task at twelve years of age. No associations between non-verbal skills at five years of age and brain activation at twelve years of age appeared in the control group.

Conclusions: The associations between better non-verbal skills and stronger neural activation during visual perception task only observed in the very preterm group, may reflect delayed development of the visual perception network and/or prematurity-related neural plasticity. The developmental follow-up of very preterm children should include psychological assessment of non-verbal skills at least until five years of age.

Keywords: prematurity; visual perception; fMRI; developmental follow-up 


\section{Introduction}

Visual perception is the process of interpreting and organizing visual information, and thus crucial in numerous tasks and situations. Difficulties with visual perception have been related to prematurity (Butcher et al. 2012; Geldof et al. 2012; Leung et al. 2018; Molloy et al. 2013) and of adolescents who were born extremely preterm and/or with an extremely low birth weight, one of four has been reported to have visual perceptual impairment (Molloy et al. 2013). Visual perceptual deficiencies have been associated with difficulties in other skills during childhood and adolescence (Leung et al. 2018; Molloy et al. 2017; Perez-Roche et al. 2016; van Veen et al. 2019). The deficiencies can be manifested, for instance, in pre-school children in gross-motor activities such as ball sports, and at school age as challenges in mathematics and reading.

Early identification of visual perceptual deficits enables interventions that could ease the difficulties and prevent later problems in areas related to visual perception. Impairments in visual perception may, however, not be detected in a routine screening of vision (Geldof et al. 2014; Leung et al. 2018). Geldof and colleagues examined visual sensory and perceptive functions and their interrelations in very preterm /very low birthweight children at five years of age. The preterm children in their study had both more visual sensory and visual perceptive deficits than term-born children, but the deficits occurred largely independently of each other. Only one third of the preterm children with perceptive deficits would have been detected in a screening relying on visual sensory measures. 
Various structural brain abnormalities and altered neural activation have been related to difficulties in visual perception, but the mechanisms underlying visual perceptual deficits in preterm children are not fully known (Geldof et al. 2012; Leung et al. 2018). Better knowledge of the factors related to visual perception could help to improve the developmental follow-up of preterm children. The aim of this study was to assess how performance in a psychological assessment of non-verbal skills at five years of age is associated with brain activation during visual perception tasks at twelve years of age in very preterm subjects without visual and neurodevelopmental impairments or major brain pathologies. It was hypothesized that test performance of the very preterm children would be poorer than in the control children, and that the non-verbal skills at five years of age would be associated with neural activation during visual perception tasks at twelve years of age in the very preterm children. 


\section{Material and methods}

\subsection{Participants}

This study is part of the multidisciplinary PIPARI project (Development and Functioning of Very Low Birth Weight Infants from Infancy to School Age). All very low birth weight ( $\leq$ 1500 g) infants born at Turku University Hospital in Finland between 2001 and 2006 who lived in the hospital catchment area and whose parents spoke and understood written Finnish or Swedish were eligible. From the beginning of 2004, the inclusion criteria were expanded to include all infants born $<32$ weeks of gestation, regardless of their birthweight. Infants with major congenital anomalies or syndromes or chromosomal anomalies were excluded. Children with known visual impairment (binocular visual acuity $<1.0$ on the Snellen scale, severe astigmatism of more than 1 diopter, amblyopic eyes or crowding phenomenon), a significant cognitive impairment (a full-scale intelligence quotient $<70$ ), cerebral palsy and/or a severe hearing impairment (loss of hearing requiring amplification in at least one ear) were also excluded from this sub-study. This sub-study includes 36 very preterm subjects born in 2004-2006 who participated in functional magnetic resonance imaging (fMRI) at twelve years of age and had data available from a psychological assessment at five years of age. An ophthalmologist examined the very preterm children at eleven years of age as a part of the developmental follow-up in the PIPARI study, and visual acuity was normal with stable fixation in all the examined subjects that met the inclusion criteria. Three of the included subjects had no data available from the ophthalmological examination. A control group of 31 term-born subjects participating in the PIPARI project, born in 2003-2004 and recruited as described in Lind et al. (2011), was included. The included control subjects had no deviating findings in structural magnetic resonance imaging (MRI) at twelve years of age. The study has been approved by the Ethical Committee of the Hospital District of Southwest 
Finland. Informed consent was obtained from the parents, and at twelve years of age, the subjects also gave their own written informed consent after receiving written information.

\subsection{Psychological assessment at five years of age}

Both the very preterm children and the controls were assessed by a psychologist within two months of their fifth birthday. Full-scale intelligence quotient (FSIQ), performance intelligence quotient (PIQ) and verbal intelligence quotient (VIQ) were estimated (M = 100, $\mathrm{SD}=15$ ) based on subtests block design, geometric design, picture completion, information, sentences and arithmetic of the Wechsler Preschool and Primary Scale of IntelligenceRevised (Wechsler, 1995). Motor and visual-perceptual skills associated with the ability to copy two-dimensional geometric figures were assessed with the design copying subtest, spatial memory for novel visual material with the memory for designs subtest and selective visual attention with the visual attention subtest from the Finnish standardization edition of the NEPSY II (Korkman et al. 2008). As the standardization edition differed somewhat from the final NEPSY II, the standard scores $(M=10, S D=3)$ used in the PIPARI study were based on the results of a control group of 149 healthy term-born children. The subtests, the control group and the testing procedure at five years of age have been described in more detail in Lind et al. (2010).

\subsection{Visual perception task at twelve years of age}

Visual perception was assessed with items from the Motor-Free Visual Perception Test, Third Edition (MVPT-3; Colarusso and Hammill, 2003) at twelve years of age. In the very preterm group, the mean age at the time of assessment was 12 years 9 months $(\mathrm{SD}=6$ months, $\min =$ 12 years 2 months, $\max =13$ years 10 months) and in the control group it was also 12 years 9 months $(\mathrm{SD}=3$ months, $\min =12$ years 2 months, $\max =13$ years 5 months $)$. An electronic 
version of the test suitable for use during fMRI, was created with permission from the publisher, Academic Therapy Publications. The items were presented on a screen during fMRI, each item had four possible answers and the participants answered by pressing one of four response buttons on a response pad. The items were presented in two series: the first series assessed visual closure, the ability to perceive a whole figure when only fragments of it are presented, and it included MVPT-3 items 22-34 and 56-60. The second series assessed discrimination of a deviating figure and included items 35-45. The stimuli were black-andwhite line drawings and designs. Each item was shown for 9 seconds and each session started with a 17-second gaze-fixation baseline period, after which the task and gaze-fixation periods alternated. Before fMRI, 1-2 training items per series were presented and the instructions were repeated orally before each series during the imaging session. The training items were not included in the actual task. In addition to brain region activation, reaction time and the number of correct responses for each series were registered. In trials where subjects performed extra button presses, the earliest response was used to define accuracy and response time. If the subject did not respond during stimulus presentation, the earliest response during the following gaze fixation period was counted. Spherical refractive errors were compensated for in the fMRI study with adjustable MRI-compatible binoculars. The visual perception scores reported are based on performance during fMRI. Five additional items from MVPT-3 assessing discrimination of figure and ground were presented during fMRI, but this fMRI series was found too short and noisy for valid fMRI analyses and therefore excluded from current study. No other psychological tests were performed at twelve years of age. 


\subsection{Brain imaging}

The MRI procedure and the classification of findings at term equivalent age and at twelve years of age are described in a supplement of the manuscript. Blood-oxygenation-leveldependent (BOLD) data was collected for 35 axial slices of $4 \mathrm{~mm}$ thickness using a singleshot sequence for $\mathrm{T} 2 *$-weighted echo planar imaging with a $2.0 \mathrm{~s}$ repetition time, a $20 \mathrm{~ms}$ echo time, a $75^{\circ}$ flip angle, an $80 \times 80$ matrix, and a $3 \mathrm{~mm}$ in-plane resolution. The first four scans of each session were removed. Each participant completed separate fMRI runs of 191231 scans for the visual closure task and of 124-147 scans for the visual discrimination task. The presentation software (https://www.neurobs.com) was used to control stimulus delivery and data collection.

\subsection{Data analyses}

For the statistical analyses of behavioral test performance, the SPSS 24.0 software (IBM, Armonk, NY, USA) was used. P-values $<0.05$ were considered statistically significant. For the comparison of test performance between the very preterm and the control group, the Mann-Whitney U-test or the independent samples t-test was used, as appropriate. For the comparison of the number of boys/girls in the groups, the Chi-Square test was used.

The MRI data were processed using Statistical Parametric Mapping (SPM12, https://www.fil.ion.ucl.ac.uk/spm) and toolboxes implemented in Matlab (MathWorks Inc., Natik MA, USA). Individual fMRI data were corrected for motion and slice-timing, and coregistered with a corresponding T1-weighed image. A customized brain template with equal contribution from both groups was created using age-specific tissue probability maps (Wilke et al. 2008) and the Computational Anatomy Toolbox CAT12 software (Gaser and Dahnke, 2016) to perform a 2-step segmentation coupled with diffeomorphic image registration 
(Ashburner, 2007). This resulted in the gray matter (GM) sample-specific template affinely registered to the Montreal Neurological Institute MNI152 space as well as a set of individual deformation fields, which were used to spatially warp the functional images into the space of the template. The functional images were also resliced to $3 \mathrm{~mm}$ cubic voxels and smoothed with an $8 \mathrm{~mm}$ Gaussian kernel.

In addition to the non-verbal variables (PIQ, design copying, memory for designs, visual attention), associations between FSIQ at five years of age and neural activation at twelve years of age were assessed. One control and one preterm participant were excluded from the fMRI analysis due to either a wide cisterna magna or a large frontal susceptibility artefact. Functional MRI data from several subjects were rejected due to motion (translation $>3 \mathrm{~mm}$ or rotation $>3^{\circ}$ ), activity related to multiple extra responses also extending to the gazefixation period, stimulus presentation problems, and missing scores from the assessment at five years of age. In the very preterm group, the number of subjects in the fMRI analyses for visual closure/visual discrimination were as follows: design copying 29/27, memory for designs 31/29, visual attention 29/27, PIQ 30/28 and FSIQ 30/28. In the control group, the number of subjects in the fMRI analyses for visual closure/visual discrimination were: design copying 25/23, memory for designs 25/23, visual attention 27/25 and PIQ 27/25. For each of the two tasks, the first-level model included a task condition of variable length epochs equal to response times (Grinband et al., 2008), condition for trials with missing response with $9 \mathrm{~s}$ duration, and implicitly modelled gaze fixation condition of $17 \mathrm{~s}$ duration. The model also included six realignment parameters, and their Euclidean norm as well as dummy regressors for outlier scans exceeding scan-to-scan changes in global mean $\mathrm{z}>4$; translation $>2 \mathrm{~mm}$; rotation $>1^{\circ}$ as provided by the Artifact Detection Tools (Susan Whitfield- Gabrieli, MIT, US). A canonical hemodynamic response function was used to convolve stimulus functions. 
Slow drifts were removed with a 128 s cut-off high-pass filter. Individual contrast images of activation differences between visual tasks and gaze fixation for each subject were fed into a second-level random-effect analysis. Correlation between each variable and the BOLD signal in either group for either task was analyzed in a separate simple regression model within a mask created by applying a 0.3 threshold to the GM template smoothed with an $8 \mathrm{~mm}$ Gaussian filter. The Threshold-Free Cluster Enhancement (TFCE; Smith and Nichols, 2009) method, as implemented in the TFCE Toolbox (Christian Gaser, Jena University Hospital, Germany), was applied to estimate a combined voxel-cluster significance using default settings, 5000 permutations and a $\mathrm{p}<0.05$ threshold for significance after family-wise error correction. 


\section{Results}

The characteristics of the very preterm children and the control group are presented in Table I. The number of boys / girls did not differ significantly $(\mathrm{p}=0.124)$ between the groups. Test scores at five and twelve years of age are shown in Table 2. Non-verbal test scores at five years of age were significantly poorer in the very preterm group than the control group, while scores at twelve years of age did not differ significantly. Exclusion of an extreme outlier in the control group did not change the significances of the results. At five years age, mean FSIQ of the very preterm children was 107 (SD 15, median 107, min 77, max 140) and of the control children 113 (SD 15, median 113, min 77, max 136). The difference was not significant. Of the very preterm children, one had FSIQ between 70 and 84 (-2 SD - -1 SD). Mean VIQ of the very preterm children was 108 (SD 14, median 109, min 77, max 130) and of the control children 109 (SD 12, median 111, $\min 77, \max 129)$, the difference was not significant. Information about received intervention following the psychological assessment at five years of age was obtained from 34 of 36 of the very preterm children. Occupational therapy had been received by $11 \%(4 / 36)$ at some point between five and eight years of age.

In the very preterm group, a higher visual attention score at the age of five was significantly associated with stronger activation in the left inferior parietal lobule, a higher memory for designs score with stronger activation in the left precuneus / posterior cingulate, and a higher design copying score with stronger activation in the right superior parietal cortex during visual discrimination task at twelve years of age (Table 3, Figure 1 and Figure 2). PIQ and FSIQ were not significantly associated with neural activation in the very preterm group. There were no significant associations between test scores at five years of age and fMRI signal at twelve years of age in the control group. 


\section{Discussion}

The aim of this study was to assess how non-verbal skills at five years of age are associated with visual perception and brain activation during visual perception tasks at twelve years of age, in very preterm subjects without visual or neurodevelopmental impairments or major brain pathologies. Non-verbal skills at five years of age were significantly poorer in the very preterm children, whereas visual perception at twelve years of age was comparable to that of the term-born control group. In the very preterm group, better visual attention, visual memory and visual-motor skills were associated with increased neural activation during the visual discrimination task in the left inferior parietal lobule, the left precuneus / posterior cingulate and the right superior parietal cortex, respectively. In the control group, no corresponding associations between test performance and neural activation appeared.

Although subjects with significant cognitive impairment and neurosensory impairments were excluded from the present study, non-verbal functions were significantly poorer in very preterm children at five years of age. Accordingly, assessment of PIQ, visual attention, visual memory and visual-motor skills should be included in the developmental follow-up of very preterm children at preschool age. Early identification of visual perceptual deficits is important as these deficits may affect various everyday activities, academic achievement and, as a result, the self-concept and self-esteem of the child. In Perez- Roche and colleagues (2016) visual perceptual skills were associated with mathematical skills and reading in children between five and thirteen years of age, born preterm or small for gestational age. Mathematical skills were particularly related to the perception of spatial relationships and discrimination of figure and ground, and reading to the perception of spatial relationships. Specific deficits in visuospatial skills at six years of age have also been shown to be predictive of mathematical and reading skills at eleven years of age in extremely preterm 
(born before gestational week 26) as well as term-born children (Johnson et al. 2011). In Johnson and colleagues, a different pattern of antecedents was observed in the preterm and term-born groups, and regarding reading, the impact of visuospatial processing appeared stronger in the group born extremely preterm. If visual perceptual deficits are identified, the children can be supported, for instance, through neuropsychological rehabilitation or occupational therapy. In some populations, also web-based programs have appeared promising in improving visual perception skills (James et al. 2015; Wan et al. 2017). For preterm infants, there is a number of intervention programs developed with the aim of preventing cognitive and motor impairments (Spittle et al. 2015), but programs specifically intended to prevent visual perceptual difficulties are lacking.

Visual perception did not differ between the very preterm and term-born groups at twelve years of age, despite the poorer non-verbal skills of the very preterm children at five years of age. A potential explanation for this is that the assessment tools used at five and twelve years of age were dissimilar, with different levels of demand and assessing different aspects of visual perceptual skills. The tasks used at twelve years of age are intended to assess visual perception, though they naturally also place demands on other functions such as working memory. Still, the tasks used at five years of age are somewhat more complex and likely require more in terms of memory functions, eye-hand coordination and attention than the MVPT-3 tasks. In a study by Wehrle and colleagues (2016), the impact of task demand on executive functions in very preterm subjects at 13 years of age was assessed. Wehrle and colleagues showed that difficulties in executive functions in very preterm adolescents were only manifested in tasks with higher demands while performance in less demanding tasks, for instance requiring less planning, were comparable with that of term-born subjects. Deficits in executive functioning in preterm children were documented also in a recent meta-analysis 
and were shown to be stable across childhood (van Houdt et al. 2019). As the tasks at five years of age in present study can be considered to require more of executive functions than the tasks at twelve years of age, it may be speculated that our results are mediated by executive functioning. It remains unclear if the performance at twelve years of age would have been comparable to that of controls if the same measures had been applied at five and at twelve years of age. It is also possible, that the visual perceptual deficits related to prematurity are more pronounced in other sub-areas of visual perception than visual closure and visual discrimination, which were assessed in the present study (Geldof et al. 2012). A third potential explanation for the lack of differences at twelve years of age is developmental catch-up. Although difficulties in visual perception have also been found to be present beyond childhood in prematurely born subjects (Geldof et al. 2012; Leung et al. 2018), there are indications that preterm children may catch up to their peers in functions related to visual perception (Murner-Lavanchy et al. 2014). In Murner-Lavanchy and colleagues, performance in a visuospatial working memory task during fMRI was comparable in preterm (born <32 weeks of gestation and/or with birth weight $<1500 \mathrm{~g}$ ) and term-born 7-12 year old children, but superior in older children compared to younger ones in both groups. In the preterm group, low-performing and younger children showed an atypical neural network, while the highperforming older children showed the typical visuospatial working memory network seen in the term-born children. Developmental catch-up in preterm subjects has been reported for other skills as well (Fitzpatrick et al. 2016; Luu et al., 2011; Reveillon et al. 2018), but there are also studies suggesting that developmental deficits related to prematurity are more persistent (Brydges et al. 2018; Linsell et al. 2018; Ngugyen et al. 2018; van Houdt et al. 2019). In present study, $11 \%$ of the very preterm children had received occupational therapy after the psychological assessment at five years of age. Hence, the potential catch-up may have been facilitated by intervention in those children. 
We are not aware of previous visual perception studies with a comparable design including fMRI. In the very preterm group, better performance in non-verbal tasks at five years of age was associated with stronger activation in the left inferior parietal lobule, the left precuneus / posterior cingulate and right superior parietal cortex during visual discrimination task at twelve years of age. No associations between performance at five years of age and later neural activation were seen in the control group, indicating that the associations found in the very preterm group reflect neural mechanisms explicitly related to prematurity. In a study by Lidzba and colleagues (2013), complex visual processing was assessed during fMRI in healthy children and adolescents at 7-17 years of age. They reported increased activation with age within the core regions of visual exploration network, mainly in right areas of the occipital and parietal cortex, while activation in other regions, such as the left inferior parietal lobe, decreased with age. Our finding of increased activation in the left inferior parietal lobule only in the very preterm group, might hence reflect delayed development of the visual processing network. Lidzba and colleagues also reported an association between better performance in the visual task and stronger activation in the right superior parietal lobe, which is in line with our results. Narberhaus and colleagues (2009) studied neural activation during a visual paired associates task in very preterm adults. In their study, the amount of correctly recognized visual pairs did not differ between the very preterm group and the control group. Brain activation during encoding in very preterm adults was, however, found to be increased compared to controls in some areas, including the left superior parietal lobule. According to Narberhaus and colleagues, their results may reflect neuroplasticity related to the neural consequences of prematurity. The cortical thickness of superior parietal regions has, in turn, been associated with visual perception in adults born with a very low birth weight (Sripada et al. 2015), and the superior parietal white matter microstructure with 
executive functioning at six years of age in a mixed group of preterm and full-term children (Dubner at el. 2019). Furthermore, the precuneus / posterior cingulate cortex has been found to interact with the left inferior parietal lobe (Fransson and Marrelec, 2008). In a study assessing neural activation differences between the recognition and encoding trials of a visual memory task, decreased activation in the precuneus / posterior cingulate cortex was displayed in very preterm born adults (Tseng et al. 2017). Also, interestingly, visual perception intervention has been shown to result in enhanced activation in superior and inferior parietal lobes in individuals with Down syndrome (Wan et al. 2017).

The small group size is a limitation of the present study as is the lack of assessment of global cognitive level at twelve years of age. Lack of information on received rehabilitation and developmental support is another restraint. When deviations appeared in the psychological assessment at five years of age, the children were directed to appropriate services and the families were instructed on how to support their child's development. The actualization of recommendations was though not followed up. A limitation is also that, of the visual perception sub-areas, only visual closure and visual discrimination were tapped with the tasks used in this study. The range of feasible tests was, however, limited as the tasks were carried out during fMRI. As previously discussed, it must also be noted that the visual perceptual tasks require other functions in addition to visual perception, and it is possible that the results are mediated by other skills. More knowledge about the developmental trajectory of visual perception in preterm children and adolescents is nonetheless needed, and the inclusion of fMRI in the present study protocol provides new insight. The very preterm study group was well-defined, and subjects with visual or neurodevelopmental impairments or major brain pathology were excluded. In future fMRI studies with preterm subjects, other sub-areas of visual perception, such as those reflecting dorsal stream functioning, should be assessed. The 
development and evaluation of early intervention for visual perception difficulties in preterm children is also important.

\section{Conclusions}

In this study, better non-verbal skills in preterm children at five years of age were associated with stronger neural activation during visual perceptual tasks at twelve years of age. These findings were specific to the very preterm children and may reflect delayed development of the visual perception network and/or neural plasticity related to prematurity. In the developmental follow-up of very preterm children, psychological assessment of visual perceptual skills is important at least until five years of age. 


\section{Acknowledgements}

The PIPARI Study Group includes, Mikael Ekblad, Satu Ekblad, Eeva Ekholm, Linda

Grönroos, Leena Haataja, Mira Huhtala, Jere Jaakkola, Max Karukivi, Pentti Kero, Riikka

Korja, Katri Lahti, Helena Lapinleimu, Liisa Lehtonen, Tuomo Lehtonen, Marika Leppänen,

Annika Lind, Mari Koivisto, Mira Mattsson, Jonna Maunu, Petriina Munck, Laura

Määttänen, Pekka Niemi, Anna Nyman, Riitta Parkkola, Liisi Ripatti, Päivi Rautava, Katriina

Saarinen, Tiina Saarinen, Susanna Salomäki, Virva Saunavaara, Sirkku Setänen, Matti

Sillanpää, Suvi Stolt, Päivi Tuomikoski, Karoliina Uusitalo and Milla Ylijoki. 


\section{References}

Ashburner J. A fast diffeomorphic image registration algorithm. Neuroimage 2007;38:95-13.

Brydges CR, Landes JK, Reid CL, Campbell C, French N, Anderson M. Cognitive outcomes in children and adolescents born very preterm: a meta-analysis. Developmental Medicine \& Child Neurology 2018;60:452-468.

Butcher PR, Bouma A, Stremmelaar EF, Bos AF, Smithson M, Van Braeckel KN.

Visuospatial perception in children born preterm with no major neurological disorders. Neuropsychology 2012;26:723-734.

Colarusso R, Hammill D. Motor-free Visual Perception Test, Third Edition. Novata, CA: Academic Therapy Publications; 2003.

Dubner SE, Dodson CK, Marchman VA, Ben-Shachar M, Feldman HM, Travis KE. White matter microstructure and cognitive outcomes in relation to neonatal inflammation in 6-yearold children born preterm. Neuroimage Clinical 2019;23:101832. [Epub ahead of print]

Fitzpatrick A, Carter J, Quigley MA. Association of Gestational Age With Verbal Ability and Spatial Working Memory at Age 11. Pediatrics 2016;138:e20160578. 
Fransson P, Marrelec G. The precuneus/posterior cingulate cortex plays a pivotal role in the default mode network: Evidence from a partial correlation network analysis. NeuroImage 2008;42:1178-1184.

Gaser G, Dahnke R. CAT - A Computational Anatomy Toolbox for the Analysis of Structural MRI Data. HBM 2016:336-348.

Geldof CJ, van Wassenaer AG, de Kieviet JF, Kok JH, Oosterlaan J. Visual perception and visual-motor integration in very preterm and/or very low birth weight children: A metaanalysis. Research in Developmental Disabilities 2012;33:726-736.

Geldof CJ, Oosterlaan J, Vuijk PJ, de Vries MJ, Kok JH, van Wassenaer-Leemhuis AG. Visual sensory and perceptive functioning in 5-year-old very preterm/very-low-birthweight children. Developmental Medicine \& Child Neurology 2014;56:862-868.

Grinband J, Wager TD, Lindquist M, Ferrera VP, Hirsch J. Detection of time-varying signals in event-related fMRI designs. Neuroimage 2008;43:509-520. 
van Houdt CA, Oosterlaan J, van Wassenaer-Leemhuis AG, van Kaam AH, AarnoudseMoens CS. Executive function deficits in children born preterm or at low birthweight: a meta-analysis. Developmental Medicine \& Child Neurology 2019;61:1015-1024.

Johnson S, Wolke D, Hennessy E, Marlow N. Educational outcomes in extremely preterm children: Neuropsychological correlates and predictors of attainment. Developmental Neuropsychology 2011;36:74-95.

James S, Ziviani J, Ware RS, Boyd RN. Randomized controlled trial of web-based multimodal therapy for unilateral cerebral palsy to improve occupational performance. Developmental Medicine \& Child Neurology 2015;57:530-538.

Korkman M, Kirk U, Kemp SL. Nepsy II - lasten neuropsykologinen tutkimus. Helsinki: Psykologien Kustannus Oy; 2008.

Leung M, Thompson B, Black J, Dai S, Alsweiler JM. The effects of preterm birth on visual development. Clinical and Experimental Optometry 2018;101:4-12.

Lidzba K, Ebner K, Hauser T-K, Wilke M. Complex visual search in children and adolescents: Effects of age and performance on fMRI activation. PLoS ONE 2013;8: e85168. 
Lind A, Haataja L, Rautava L, Väliaho A, Lehtonen L, Lapinleimu H, Parkkola R, Korkman M and the PIPARI Study Group. Relations between brain volumes, neuropsychological assessment and parental questionnaire in prematurely born children. European Child \& Adolescent Psychiatry 2010;19:407-417.

Lind A, Korkman M, Lehtonen L, Lapinleimu H, Parkkola R, Matomäki J, Haataja L and the PIPARI Study Group. Cognitive and neuropsychological outcomes at 5 years of age in preterm children born in the 2000s. Developmental Medicine \& Child Neurology 2011;53:256-262.

Linsell L, Johnson S, Wolke D, O'Reilly H, Morris JK, Kurinczuk JJ, Marlow N. Cognitive trajectories from infancy to early adulthood following birth before 26 weeks of gestation: a prospective, population-based cohort study. Archives of Disease in Childhood 2018;103:363370.

Luu TM, Vohr BR, Allan W, Schneider KC, Ment LR. Evidence for catch-up in cognition and receptive vocabulary among adolescents born very preterm. Pediatrics 2011;128:313-22.

Molloy CS, Wilson-Ching M, Anderson VA, Roberts G, Anderson PJ, Doyle LW. Visual processing in adolescents born extremely low birth weight and/or extremely preterm. Pediatrics 2013;132:e704-712. 
Molloy CS, Di Battista AM, Anderson VA, Burnett A, Lee KJ, Roberts G, Cheong JL, Anderson PJ, Doyle LW. The contribution of visual processing to academic achievement in adolescents born extremely preterm or extremely low birth weight. Child Neuropsychology $2017 ; 23: 361-379$

Murner-Lavanchy I, Ritter BC, Spencer-Smith MM, Perrig WJ, Schroth G, Steinlin M, Everts R. Visuospatial working memory in very preterm and term born children-impact of age and performance. Developmental Cognitive Neuroscience 2014;9:106-116.

Narberhaus A, Lawrence E, Allin M P, Walshe M, McGuire P, Rifkin L, Murray R, Nosarti C . Neural substrates of visual paired associates in young adults with a history of very preterm birth: Alterations in fronto-parieto-occipital networks and caudate nucleus. Neuroimage 2009;47:1884-1893.

Nguyen TN, Spencer-Smith M, Zannino D, Burnett A, Scratch SE, Pascoe L, Ellis R, Cheong J, Thompson D, Inder T, Doyle LW, Anderson PJ. Developmental Trajectory of Language From 2 to 13 Years in Children Born Very Preterm. Pediatrics 2018;141:e20172831.

Perez-Roche T, Altemir I, Gimenez G, Prieto E, Gonzalez I, Pena-Segura JL, Castillo O, Pueyo V. Effect of prematurity and low birth weight in visual abilities and school performance. Research in Developmental Disabilities 2016;59:451-457. 
Réveillon M, Hüppi PS, Barisnikov K. Inhibition difficulties in preterm children:

Developmental delay or persistent deficit? Child Neuropsychology 2018;24:734-762.

Smith SM, Nichols TE. Threshold-free cluster enhancement: addressing problems of smoothing, threshold dependence and localisation in cluster inference. Neuroimage 2009;44:83-98.

Spittle A, Orton J, Anderson PJ, Boyd R, Doyle LW. Early developmental intervention programmes provided post hospital discharge to prevent motor and cognitive impairment in preterm infants. Cochrane Database of Systematic Reviews 2015;11:CD005495.

Sripada K, Løhaugen GC, Eikenes L, Bjørlykke KM, Håberg AK, Skranes J, Rimol LM. Visual-motor deficits relate to altered gray and white matter in young adults born preterm with very low birth weight. Neuroimage 2015;109:493-504.

Tseng CJ, Froudist-Walsh S, Brittain PJ, Karolis V, Caldinelli C, Kroll J, Counsell SJ, Williams SCR, Murray RM, Nosarti C. A Multimodal Imaging Study of Recognition Memory in Very Preterm Born Adults. Human Brain Mapping 2017;38:644-655.

van Veen S, van Wassenaer-Leemhuis AG, van Kaam AH, Oosterlaan J, Aarnoudse-Moens CSH. Visual perceptive skills account for very preterm children's mathematical difficulties in preschool. Early Human Development 2019;129:11-15. 
Wan YT, Chiang CS, Chen SC, Wuang YP. The effectiveness of the computerized visual perceptual training program on individuals with Down syndrome: An fMRI study. Research in Developmental Disabilities 2017;66:1-15.

Wechsler D. Wechsler Preschool and Primary Scale of Intelligence-Revised. Helsinki: Psykologien Kustannus Oy; 1995.

Wehrle FM, Kaufmann L, Benz LD, Huber R, O'Gorman RL, Latal B, Hagmann CF. Very preterm adolescents show impaired performance with increasing demands in executive function tasks. Early Human Development 2016;92:37-43

Wilke M, Holland SK, Altaye M, Gaser C. Template-O-Matic: a toolbox for creating customized pediatric templates. Neuroimage 2008;41:903-913. 


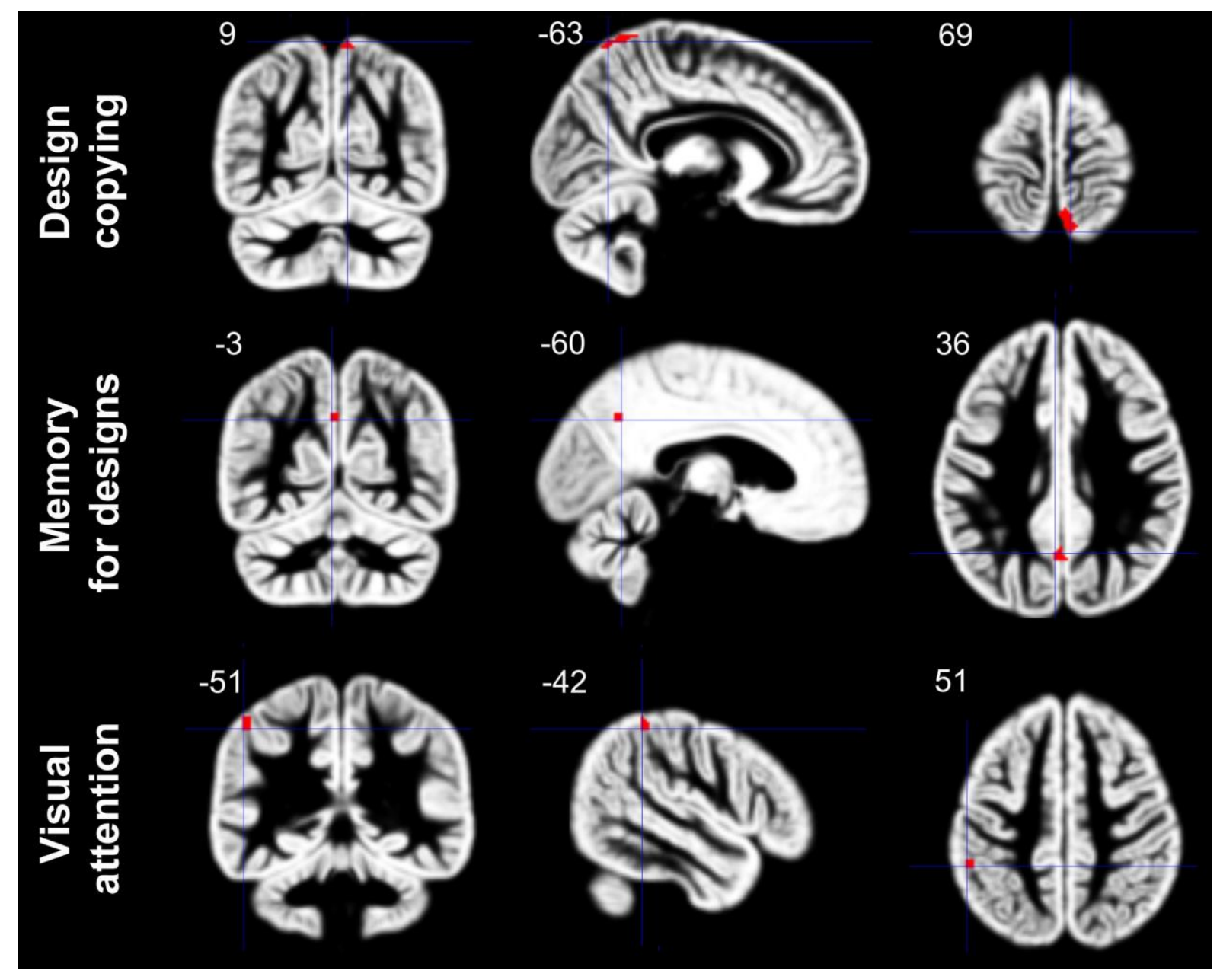

Figure 1. Areas of significant positive correlation between design copying (first row, $\mathrm{n}=27$ ), memory for designs (second row, $\mathrm{n}=29$ ) and visual attention (third row, $\mathrm{n}=27$ ) and neural activation during visual discrimination in the very preterm group. The areas are shown overlaid onto three orthogonal planes of the sample-specific template crossing the cluster maxima, indicated by cross-hairs and Montreal neurological institute coordinates next to each section. 

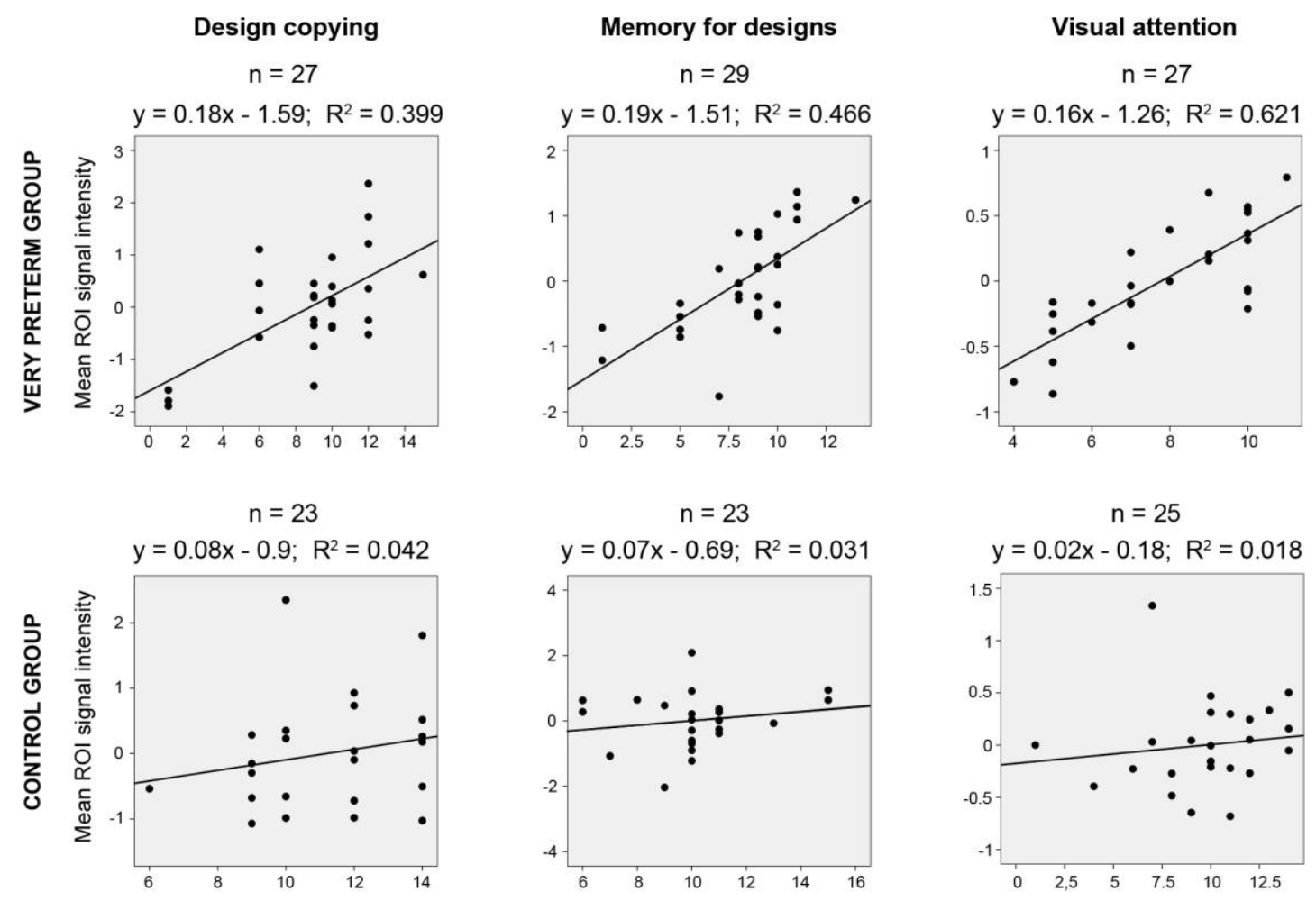

Figure 2. Scatterplots and linear regression lines for dependencies of adjusted mean region of interest (ROI) intensities in the very preterm (upper row) and control (bottom row) group on subtests design copying (left column), memory for designs (central column), and visual attention (right column). Regression parameters and coefficient of determination $\left(\mathrm{R}^{2}\right)$ are indicated above each plot. The ROIs represented clusters surviving family-wise error corrected $\mathrm{p}<0.05$ threshold based on the threshold-free cluster enhancement statistics. 
Table 1. Background characteristics of the very preterm and the control group.

$\begin{array}{ll}\text { Very preterm group } & \text { Control group } \\ \mathrm{n}=36 & \mathrm{n}=31\end{array}$

Gestational age, weeks, mean (SD) min-max

Birth weight, g, mean (SD) min-max

Boys / girls, n (\%)

Major brain pathology at term equivalent age, $\mathrm{n}(\%)$

Major brain pathology at age twelve years, $\mathrm{n}(\%)$
29 (3) $24-33$

1133 (309) 620-1970

$23(64 \%) / 13(36 \%)$

$1(3 \%)$

$0(0 \%)$
40 (1) $37-41$

3621 (489) 2830-4580

$14(45 \%) / 17(55 \%)$

$0(0 \%)$

Data on brain pathology at term age was missing for one preterm subject. 
Table 2. Test results at five and twelve years of age and the differences between the very preterm group and the control group.

\begin{tabular}{lll}
\hline Very preterm group & Control group & P-value \\
$\mathrm{n}=36$ & $\mathrm{n}=31$ \\
\hline
\end{tabular}

Assessment at five years of age

Performance intelligence quotient

mean (SD) median, min-max

Design copying

mean (SD) median, min-max

Memory for designs

mean (SD) median, min-max
104 (14) 104, 74-136

112 (14) $113,84-135$

0.027
$10.9(2.3) 10,6-14$

0.013

Visual attention

$7.9(2.9) 9,1-14 \quad 9.8(2.5) 10,4-15$

0.005 
Assessment at twelve years of age

Visual closure, accuracy

mean (SD) median, min-max

$12.8(2.2) 13,7-16$

$12.8(3.3) 14,0-17$

0.379

Visual closure, response time

mean (SD) median, min-max

$5.0(1.0) 4.9,2.8-8.5$

4.9 (1.1) 4.9, 3.0-7.5

0.844

Visual discrimination, accuracy

mean (SD) median, min-max

$6.2(1.6) 6,2-9$

6.3 (1.7) 7, 0-9

0.698

Visual discrimination, response time

mean (SD) median, min-max
$4.8(0.9) 4.6,3.0-7.0$

$4.4(1.0) 4.3,2.7-6.8$

0.082 
Data on performance intelligence quotient was missing for one preterm subject, data on visual attention for two, data on design copying for two and data on visual closure accuracy/response time for one. Data on memory for designs was missing for two control subjects and data on design copying for two. One control subject who did not give any correct responses in the visual closure and discrimination of a deviating figure tasks was not included in the analyses of response time of correct responses. P-values $<0.05$ were considered significant. 
Table 3. Associations between performance in non-verbal subtests at five years of age and neural activation during visual discrimination task at twelve years of age in the very preterm group.

\begin{tabular}{|c|c|c|c|c|c|c|c|c|}
\hline \multirow[t]{2}{*}{$\mathrm{n}$} & \multirow[t]{2}{*}{ Subtest } & \multirow[t]{2}{*}{ Area } & \multirow[t]{2}{*}{ Cluster size } & \multirow{2}{*}{$\begin{array}{l}\text { P-value } \\
\text { (FWE) }\end{array}$} & \multirow[t]{2}{*}{ TFCE value } & \multicolumn{3}{|c|}{ MNI coordinates } \\
\hline & & & & & & $\mathrm{x}$ & $\mathrm{y}$ & $\mathrm{z}$ \\
\hline 27 & Design copying & Right superior parietal lobule & 25 & 0.031 & 497.39 & 9 & -63 & 69 \\
\hline 29 & Memory for designs & Left precuneus / posterior cingulate & 10 & 0.039 & 474.67 & -3 & -60 & 36 \\
\hline 27 & Visual attention & Left inferior parietal lobule & 3 & 0.045 & 444.22 & -51 & -42 & 51 \\
\hline
\end{tabular}

FWE $=$ family-wise error corrected for multiple comparisons, TFCE $=$ threshold-free cluster enhancement, MNI $=$ Montreal Neurological Institute 
